\title{
Global Optimization of Multivariate Holderian Functions Using Overestimators
}

\author{
Amine Yahyaoui', Hamadi Ammar ${ }^{2}$ \\ ${ }^{1}$ Faculty of Sciences of Bizerte, Carthage University, Tunis, Tunisia \\ ${ }^{2}$ Faculty of Economics and Management of Nabeul, Carthage University, Tunis, Tunisia \\ Email: AmineYahyaoui1919@Gmail.com,Hamadi.Ammar63@Gmail.com
}

How to cite this paper: Yahyaoui, A. and Ammar, H. (2017) Global Optimization of Multivariate Holderian Functions Using Overestimators. Open Access Library Journal, 4: e3511.

https://doi.org/10.4236/oalib.1103511

Received: March 9, 2017

Accepted: May 2, 2017

Published: May 5, 2017

Copyright $\odot 2017$ by authors and Open Access Library Inc.

This work is licensed under the Creative Commons Attribution International License (CC BY 4.0).

http://creativecommons.org/licenses/by/4.0/

(c) (i) Open Access

\begin{abstract}
This paper deals with the global optimization of several variables Holderian functions. An algorithm using a sequence of overestimators of a single variable objective function was developed converging to the maximum. Then by the use of $\alpha$-dense curves, we show how to implement this algorithm in a multidimensional optimization problem. Finally, we validate the algorithm by testing it on some test functions.
\end{abstract}

\section{Subject Areas}

Numerical Mathematics, Operational Research

\section{Keywords}

Global Optimization, Branch and Bound, Holderian Functions, Alienor Method

\section{Introduction}

When modeling economic, biologic, ..., systems, we often meet situations where we are led to minimize or maximize objective multivariate functions [1]. Generally, we are seeking global optimums. It's well known that global optimization algorithms are scare, when compared to the local optimization ones [2], and when they exist, their implementation is not so obvious. This difficulty increases when the number of the decision variables gets higher.

In this paper, the objective function is deterministic and available and the variables are bounded but the derivative information is either unavailable or its manipulation is expensive.

When information derivative is not required, many authors have used the regularity of the objective function to elaborate algorithms giving the optimum [3] [4]. 
Shubert [5], Ammar and Cherruault [6] [7], Evtushenko Ya. G., Malkova V. U. and Stanevichyus A. A. [8], Gergel V. P. and Sergeyev Ya. D. [9], Sergeyev Y. D. and Kvasov D. E. [10] considered the case where the objective function is lipschitzian. They developed methods generating sequences converging to the optimum. Other authors, Gourdin E. Jaumard B. and Ellaia R. [11], Lera D. and Sergeyev Ya. D. [12], Rahal M. and Ziadi A. [13], processed the case of holderian functions by trying to elaborate a sequence to converge to the optimum; except that, here, obtaining a sequence, to converge to the optimum, is not so obvious.

In this paper, we are also interested in holderian objective functions. We will develop a technique to solve a multidimensional optimization problem.

In the first part of this paper we define a sequence of overestimators of a single variable function. Then we describe a global optimization algorithm suitable to such functions converging to the global maximum. Then after, we show how we can give an approximating value of the maximum of a several-variables holderian function. To do this, we introduce, in the second part, the Lissajous $\alpha$-dense curve: the tool that allows to go from a multidimensional optimization problem to a single dimensional one. We end this paper by validating our algorithm testing it on some test functions [14].

\section{Optimization of a Single Variable Hoderian Function}

Let's consider a single variable holderian function $f$ defined on an interval $[a, b] \subset \mathbb{R}$.

Let's denote by $(\mathrm{P})$ the following unidimensional optimization problem:

$$
\text { (P) }\left\{\begin{array}{l}
\text { Maximize } f(x) \\
x \in[a, b]
\end{array}\right.
$$

In fact, we will not search the exact solution $x_{o p t}$ of this problem, we just want to have its approximated value. To achieve this, we will develop a global optimization algorithm suited to holderian functions, that will give an approximation $x^{*}$ such that $\left|f\left(x_{o p t}\right)-f\left(x^{*}\right)\right| \leq \varepsilon_{0}$ where $\varepsilon_{0}>0$, is the required accuracy a priori chosen. This algorithm is based on a sequence of overestimators.

\subsection{Overestimator of a Holderian Function}

Definition 1. A real multivariate function $f$ is said to be holderian on a set $X \subset \mathbb{R}^{n}$, if there exists $k>0$ and $\beta>1$ such that $\forall x \in X$ and $y \in X$ :

$$
|f(x)-f(y)| \leq k|x-y|^{\frac{1}{\beta}} \text {. }
$$

Definition 2. A function $F$ is said to be an overestimator of a function $f$ on a set $X$ if:

$$
\forall x \in X, F(x) \geq f(x) \text {. }
$$

Proposition 1. Let $f$ be a holderian univariate function defined on the interval $[a, b]$ and let $y \in[a, b]$. The function $H$ defined on $[a, b]$ by: $\forall x \in[a, b]$

$$
H(x)=f(y)+k|x-y|^{\frac{1}{\beta}} .
$$


is an overestimator of $f$ on $[a, b]$.

Proof. Let's set $y \in[a, b]$, As $f$ is holderian: $\forall x \in[a, b]$

$$
|f(x)-f(y)| \leq k|x-y|^{\frac{1}{\beta}} .
$$

This yields: $f(x)-f(y) \leq k|x-y|^{\frac{1}{\beta}}$.

Hence, $f(x) \leq f(y)+k|x-y|^{\frac{1}{\beta}}=H(x)$.

\subsection{Sequence of Overestimators}

Let $x_{0}=a$ the left bound of $[a, b]$ and let's set:

$$
F_{0}(x)=f\left(x_{0}\right)+k\left|x-x_{0}\right|^{\frac{1}{\beta}}=G_{0}(x)
$$

an overestimator of $f$ whose representative curve is given by Figure 1 .

The curve has one vertex $V_{1}\left(u_{1}=b, H_{1}\right)$ such that:

$$
H_{1}=f\left(x_{0}\right)+k\left|b-x_{0}\right|^{\frac{1}{\beta}}=\max _{x \in[a, b]} G_{0}(x)
$$

Let's set $x_{1}=\arg \max \left(G_{0}(x)\right)$. Here, $x_{1}=b$. From the point that coordinates are $\left(x_{1}, f\left(x_{1}\right)\right)$, we plot the curve of the overestimator:

$$
F_{1}(x)=f\left(x_{1}\right)+k\left|x-x_{1}\right|^{\frac{1}{\beta}}
$$

as shown in Figure 2. We set:

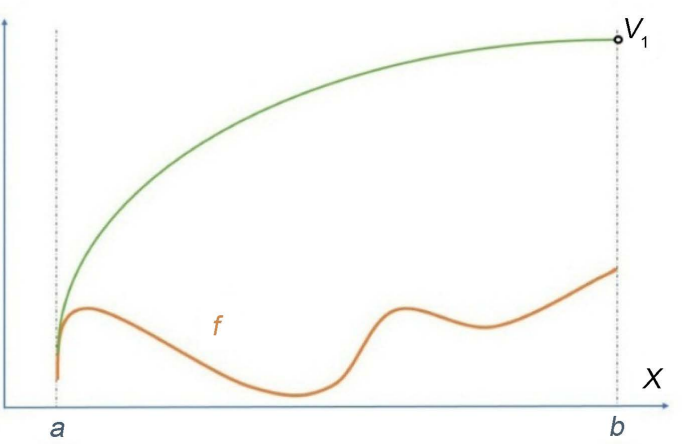

Figure 1. Curve of $F_{0}$.

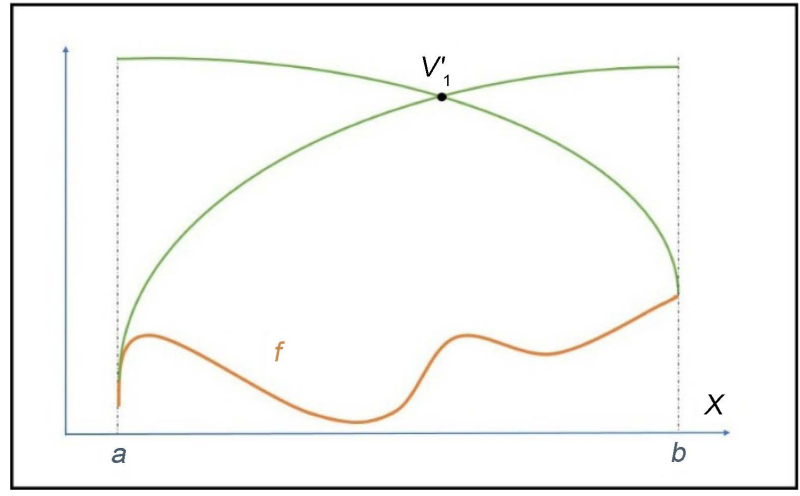

Figure 2. Curve of $G_{1}$. 


$$
G_{1}(x)=\min \left(F_{1}(x), G_{0}(x)\right)
$$

and $x_{2}=\arg \max \left(G_{1}(x)\right)$.

The vertex $V_{1}$ is anymore a vertex of the curve of $G_{1}$. It's replaced by a new vertex $V_{1}^{\prime}\left(x_{2}, G_{1}\left(x_{2}\right)\right)$, given by the intersection of the curves of $G_{1}$ and $F_{1}$.

The real $x_{2}$ is solution of the following equation:

$$
f\left(x_{0}\right)+k\left|x_{2}-x_{0}\right|^{\frac{1}{\beta}}=f\left(x_{1}\right)+k\left|x_{2}-x_{1}\right|^{\frac{1}{\beta}}
$$

In general, it is not easy to have the exact value of the solution of the equation above. For this reason, we will introduce an auxiliary function $O_{1}$ that allows to give a value nearby to $x_{2}$ that we also denote by $x_{2}$.

The point $V_{1}^{\prime}\left(x_{2}, G_{1}\left(x_{2}\right)\right)$ is between two neighbouring points belonging to the curve of $G_{1}$ : one on its left $L\left(x_{0}, f\left(x_{0}\right)\right)$ and one in its right $R\left(x_{1}, f\left(x_{1}\right)\right)$. We denote by:

- $M_{1}=\max \left(f\left(x_{0}\right), f\left(x_{1}\right)\right)$

- $m_{1}=\min \left(f\left(x_{0}\right), f\left(x_{1}\right)\right)$

- $\mu_{1}=\arg \max \left(f\left(x_{0}\right), f\left(x_{1}\right)\right)$

- $\rho_{1}=\arg \min \left(f\left(x_{0}\right), f\left(x_{1}\right)\right)$

According to the Figure 2, and in this case, $M_{1}=f\left(x_{1}\right)$ and $m_{1}=f\left(x_{0}\right)$. Let's set $z_{1}$ in $\left[x_{0}, x_{1}\right]$ such that: $G_{1}\left(z_{1}\right)=M_{1}$ and $z_{1} \neq \mu_{1}$. That yields that:

$$
z_{1}=x_{0}+\left(\frac{M_{1}-m_{1}}{k}\right)^{\beta}
$$

From the point $L_{1}\left(z_{1}, M_{1}\right)$, we plot the representative curve of:

$$
O_{1}(x)=\min \left(M_{1}+k\left|x-x_{1}\right|^{\frac{1}{\beta}}, M_{1}+k\left|x-\mu_{1}\right| \frac{1}{\beta}\right) 1_{J_{1}}(x)
$$

where $J_{1}=\left[\min \left(z_{1}, \mu_{1}\right), \max \left(z_{1}, \mu_{1}\right)\right]$.

The new function:

$$
G_{1}^{\prime}(x)=G_{1}(x) 1_{x \notin J_{1}}+O_{1}(x)
$$

is also an overestimator.

The curve of $G_{1}^{\prime}$ has a new vertex, given by the curve of $O_{1}$, denoted by: $V^{\prime \prime}\left(u_{1}, H_{1}^{\prime}\right)$ such that: $\left\{\begin{array}{l}H_{1}^{\prime}=M_{1}+k\left|u_{1}-z_{1}\right|^{\frac{1}{\beta}} \\ u_{1}=\frac{z_{1}+x_{1}}{2}\end{array}\right.$ as indicated in Figure 3.

Hence, the vertex $V_{1}$ will be replaced by $V^{\prime \prime}$. The new vertex of the curve of $G_{1}^{\prime}$, now denoted by $V_{1}$, will be identified by $\left(u_{1}, L_{1}, R_{1}, H_{1}\right)$ with $H_{1}=M_{1}+k\left|u_{1}-z_{1}\right|^{\frac{1}{\beta}}$ and where $L_{1}\left(z_{1}, M_{1}\right)$ and $R_{1}\left(x_{1}, M_{1}\right)$ are, respectively, the left and the right neighbours of $V_{1}$.

Let set $x_{2}=\arg \max \left(G_{1}^{\prime}(x)\right)$. Here, $x_{2}=u_{1}$. Let:

- $F_{2}(x)=f\left(x_{2}\right)+k\left|x-x_{2}\right|^{\frac{1}{\beta}}$ 
- $G_{2}(x)=\min \left(F_{2}(x), G_{1}^{\prime}(x)\right)$

Suppose $f$ evaluated at $x_{0}, x_{1}, \cdots, x_{n}$ and denote by:

$$
\varphi_{n}=\max \left(f\left(x_{0}\right), f\left(x_{1}\right), \cdots, f\left(x_{n}\right)\right)
$$

The curve of $G_{n}^{\prime}$ has $n$ vertexes: $V_{0} \leq V_{1} \leq \cdots \leq V_{n}$ such that each of them is identified by:

$$
\left\{\begin{array}{l}
\text { its left neighbour } L_{i}\left(l_{i}, M_{i}\right) \\
\text { its right neighbour } R_{i}\left(r_{i}, M_{i}\right) \\
\text { its absciss } u_{i}=\frac{l_{i}+r_{i}}{2} \\
\text { its ordinate } H_{i}=M_{i}+k\left|u_{i}-r_{i}\right|^{\frac{1}{\beta}}
\end{array} \text { for } i \text { from } 1 \text { to } n .\right.
$$

Let's $x_{n+1}=\arg \max \left(G_{n}^{\prime}(x)\right)=u_{n}, \quad y_{n+1}=f\left(x_{n+1}\right), \quad \varphi_{n+1}=\max \left(\varphi_{n}, f\left(x_{n+1}\right)\right)$ and:

- $F_{n+1}(x)=f\left(x_{n+1}\right)+k\left|x-x_{n+1}\right|^{\frac{1}{\beta}}$

- $G_{n+1}(x)=\min \left(G_{n}^{\prime}(x), F_{n+1}(x)\right)$

For the curve of the overestimator $G_{n+1}, V_{n}$ is anymore a summit, but two new vertexes appear from either side of $V_{n}$ : denoted by $V_{L}$ (in the left) and $V_{R}$ (in the right), as indicated in Figure 4. Set:

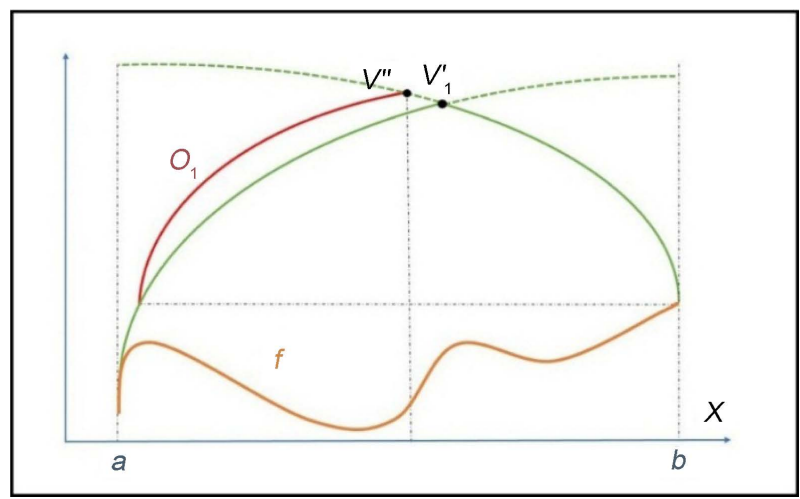

Figure 3. Curve of $G_{1}^{\prime}$.

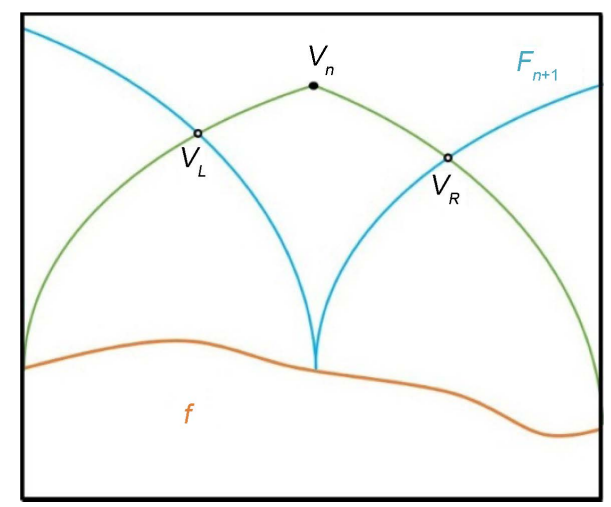

Figure 4. Curve of $G_{n+1}$. 
- $M_{n+1}=\max \left(f\left(x_{n+1}\right), M_{n}\right)$

- $m_{n+1}=\min \left(f\left(x_{n+1}\right), M_{n}\right)$

For the both vertexes $V_{L}$ and $V_{R}$, it is not obvious to calculate their coordinates. Each of them will be replaced, respectively, by $V_{L}^{\prime}$ and $V_{R}^{\prime}$ as proceeded for $G_{1}$.

Let's determinate the coordinates of vertex $V_{L}^{\prime}$.

Set $\mu_{n+1}^{L}=\arg \left(M_{n+1}\right)$ and $\rho_{n+1}^{L}=\arg \left(m_{n+1}\right)$ which belong to the set $\left\{x_{n+1}, l_{n}\right\}$ where $l_{n}$ is the absciss of the left neighbour $L_{n}$ of $V_{n}$, as mentioned in (1).

Let's set $z_{L}$ in $\left[x_{n+1}, \mu_{n+1}^{L}\right]$ such that: $G_{n+1}\left(z_{L}\right)=M_{n+1}$ and $z_{L} \neq \mu_{n+1}^{L}$.

This involves:

$$
z_{L}=\rho_{n+1}^{L}+\operatorname{sign}\left(\mu_{n+1}^{L}-\rho_{n+1}^{L}\right)\left(\frac{M_{n+1}-m_{n+1}}{k}\right)^{\frac{1}{\beta}}
$$

where $\operatorname{sign}(x)=\left\{\begin{array}{l}+1, \text { if } x \geq 0 \\ -1, \text { if } x<0\end{array}\right.$. Let:

$$
O_{n+1}^{L}(x)=\min \left(M_{n+1}+k\left|x-z_{L}\right|^{\frac{1}{\beta}}, M_{n+1}+k\left|x-\mu_{n+1}^{L}\right|^{\frac{1}{\beta}}\right) 1_{J_{n+1}^{L}}(x)
$$

where $J_{n+1}^{L}=\left[\min \left(z_{L}, \mu_{n+1}^{L}\right), \max \left(z_{L}, \mu_{n+1}^{L}\right)\right]$.

The part of the curve of $G_{n+1}$ relative to the interval $\left[\min \left(z_{L}, \mu_{n+1}^{L}\right), \max \left(z_{L}, \mu_{n+1}^{L}\right)\right]$ is replaced by the one of $O_{n+1}^{L}$. That makes appear a new vertex $\left(V_{L}^{\prime}\right)$ replacing $V_{L}$ such that:

- Its absciss is $u_{L}=\frac{1}{2}\left(z_{L}+\mu_{n+1}^{L}\right)$

- Its ordinate is $H_{L}=M_{n+1}+k\left|\mu_{n+1}^{L}-u_{L}\right|^{\frac{1}{\beta}}$

Furthermore, $V_{L}^{\prime}$ will be identified by its neighbours:

- The left neighbour $L\left(\min \left(z_{L}, \mu_{n+1}^{L}\right), M_{n+1}\right)$

- The right neighbour $R\left(\max \left(z_{L}, \mu_{n+1}^{L}\right), M_{n+1}\right)$

Those values will be saved in memory.

Similarly, $V_{R}$ will be replaced by $V_{R}^{\prime}$ determined as follows:

Set $\mu_{n+1}^{R}=\arg \left(M_{n+1}\right)$ and $\rho_{n+1}^{R}=\arg \left(m_{n+1}\right)$ which belong to the set $\left\{x_{n+1}, r_{n}\right\}$ where $r_{n}$ is the absciss of the right neighbour of $V_{n}$. Let:

$$
\begin{aligned}
& \checkmark \quad z_{R}=\rho_{n+1}^{R}+\operatorname{sign}\left(\mu_{n+1}^{R}-\rho_{n+1}^{R}\right)\left(\frac{M_{n+1}-m_{n+1}}{k}\right)^{\frac{1}{\beta}} \\
& \checkmark \quad O_{n+1}^{R}(x)=\min \left(M_{n+1}+k\left|x-z_{R}\right|^{\frac{1}{\beta}}, M_{n+1}+k\left|x-\mu_{n+1}^{R}\right|^{\frac{1}{\beta}}\right) 1_{J_{n+1}^{R}}(x)
\end{aligned}
$$

where $J_{n+1}^{R}=\left[\min \left(z_{R}, \mu_{n+1}^{R}\right), \max \left(z_{R}, \mu_{n+1}^{R}\right)\right]$.

The vertex $V_{R}^{\prime}$ that will replace $V_{R}$ has the following coordinates: 
- Its absciss is $u_{R}=\frac{1}{2}\left(z_{R}+\mu_{n+1}^{R}\right)$

- Its ordinate is $H_{R}=M_{n+1}+k\left|\mu_{n+1}^{R}-u_{R}\right|^{\frac{1}{\beta}}$ $V_{R}^{\prime} \quad$ will also be identified by its neighbours:

- The left neighbour $L\left(\min \left(z_{R}, \mu_{n+1}^{R}\right), M_{n+1}\right)$

- The right neighbour $R\left(\max \left(z_{R}, \mu_{n+1}^{R}\right), M_{n+1}\right)$

Let's set:

$$
G_{n+1}^{\prime}(x)=G_{n+1}^{L}(x)+G_{n+1}^{R}(x)+G_{n+1}(x) 1_{x \in J_{n+1}}(x)
$$

where $J_{n+1}=\left[\min \left(z_{L}, \mu_{n+1}^{L}\right), \max \left(z_{R}, \mu_{n+1}^{R}\right)\right]=J_{n+1}^{L} \cup J_{n+1}^{R}$.

Furthermore, the vertex $V_{n}$ is eliminated and replaced by $V_{R}^{\prime}$ and $V_{L}^{\prime}$. Hence, we have $n+1$ simmits that we organize in an increasing order, that yields:

$$
V_{1} \leq V_{2} \leq \cdots \leq V_{n+1}
$$

\subsection{Convergence Theorem}

Theorem 1. Let $f$ a $\left(C, \frac{1}{\beta}\right)$-holderian function defined on the interval $[a, b]$. The sequence $\left(H_{n}\right)_{n \in \mathbb{N}}$, defined above, decreases to the maximum of $f$.

Proof. Denote by $\varphi=\max _{x \in[a, b]} f(x)$ and $\Phi=\{x \in[a, b] ; f(x)=\varphi\}$, $\forall x \in[a, b], G_{n}(x) \geq f(x)$ for all $n \in \mathbb{N}$. This involves that:

$$
H_{n}=\max _{x \in[a, b]} G_{n}(x) \geq \max _{x \in[a, b]}(x)=\varphi
$$

As $\varphi_{n+1}=\max \left(f\left(x_{0}\right), \cdots, f\left(x_{n+1}\right)\right)$ and by the construction of $M_{n}$, we deduce that:

$$
M_{n+1} \leq \varphi_{n+1} \leq H_{n+1}
$$

Hence:

$$
\begin{aligned}
\left|H_{n+1}-\varphi_{n+1}\right| & \leq\left|H_{n+1}-M_{n+1}\right| \leq k\left|x_{L_{n+1}}-x_{n+1}\right|^{\frac{1}{\beta}} \leq k\left|\frac{1}{2}\left(x_{L_{n+1}}-x_{R_{n+1}}\right)\right|^{\frac{1}{\beta}} \\
& \leq k\left|\frac{1}{2^{2}}\left(x_{L_{n}}-x_{R_{n}}\right)\right|^{\frac{1}{\beta}} \leq k\left|\frac{1}{2^{n}}\left(x_{L_{1}}-x_{R_{1}}\right)\right|^{\frac{1}{\beta}}
\end{aligned}
$$

which vanishes to 0 .

As $\left(\varphi_{n}\right)$ is an increasing bounded sequence, it converges. Suppose that $\varphi_{n}$ converges to $\mu \neq \varphi$. As $f([a, b])$ is a compact, $\mu \in f([a, b])$. Let $y_{n} \in[a, b]$ such that $\varphi_{n}=f\left(y_{n}\right)$. As $[a, b]$ is a compact, the sequence $\left(y_{n}\right)$ admits a subsequence $\left(y_{n_{k}}\right)$ that converges to $z$ in $[a, b]$. The continuity of $f$ involves that $f(z)=\mu$.

Let $\varepsilon=\varphi-\mu$. Since $\left(y_{n_{k}}\right)$ converges to $z, \exists K, \forall k \geq K,\left|y_{n_{k}}-z\right|<\left(\frac{\varepsilon}{2 C}\right)^{\beta}$. 
The property of Holder involves that: $\left|f\left(y_{n_{k}}\right)-f(z)\right| \leq C\left|y_{n_{k}}-z\right|^{\frac{1}{\beta}} \leq \frac{\varepsilon}{2}$. This means that $\forall k \geq K$ :

$$
\varphi_{n_{k}}=f\left(y_{n_{k}}\right) \leq f(z)+\frac{\varepsilon}{2}=\mu+\frac{\varepsilon}{2} .
$$

On the other hand, $\forall n \geq n_{k}$ :

$$
G_{n}(x) \leq G_{n_{k}}(x)=f\left(y_{n_{k}}\right)+C\left|x-y_{n_{k}}\right|^{\frac{1}{\beta}}=\varphi_{n_{k}}+C\left|x-y_{n_{k}}\right|^{\frac{1}{\beta}} .
$$

Hence, $\forall x \in[a, b]$ and $\forall n \geq n_{k}$ such that $\left|x-y_{n_{k}}\right|<\left(\frac{\varepsilon}{2 C}\right)^{\beta}$, we have:

$$
G_{n}(x)=\varphi_{n_{k}}+\frac{\varepsilon}{2} \leq \mu+\frac{\varepsilon}{2}+\frac{\varepsilon}{2}=\varphi
$$

The real $z \in] y_{n_{k}}-\left(\frac{\varepsilon}{2 C}\right)^{\beta}, y_{n_{k}}+\left(\frac{\varepsilon}{2 C}\right)^{\beta}[$, then $\exists j>K$ such that:

$$
\left|y_{n_{j}}-y_{n_{K}}\right|<\left(\frac{\varepsilon}{2 C}\right)^{\beta} \text {. }
$$

This means that $H_{n_{j}}=G_{n_{j}}\left(y_{n_{j}}\right)<\varphi$. This is absurd. Then $\left(y_{n_{k}}\right)$ converges to $\varphi$.

\subsection{Description of the Algorithm}

\subsubsection{Initialization}

$$
x_{0}=a, x_{1}=b, n=1 .
$$

- $M_{1}=\max \left(f\left(x_{0}\right), f\left(x_{1}\right)\right)$

- $m_{1}=\min \left(f\left(x_{0}\right), f\left(x_{1}\right)\right)$

- $\mu_{1}=\arg \max \left(f\left(x_{0}\right), f\left(x_{1}\right)\right)$

- $V_{1}\left(u_{1}=\frac{z_{1}+x_{1}}{2}, H_{1}=M_{1}+k\left|u_{1}-z_{1}\right|^{\frac{1}{\beta}}\right)$

$$
L_{1}\left(z_{1}, M_{1}\right) \text { and } R_{1}\left(x_{1}, M_{1}\right)
$$

\subsubsection{Iterative Steps}

- $\varphi_{n}=\max \left(f\left(x_{0}\right), f\left(x_{1}\right), \cdots, f\left(x_{n}\right)\right)$

- $x_{n+1}=u_{n}$

- $M_{n+1}=\max \left(f\left(x_{n+1}\right), M_{n}\right)$

- $m_{n+1}=\min \left(f\left(x_{n+1}\right), M_{n}\right)$

- $V_{L}^{\prime}\left\{\begin{array}{l}u_{L}=\frac{1}{2}\left(z_{L}+\mu_{n+1}^{L}\right) \\ H_{L}=M_{n+1}+k\left|\mu_{n+1}^{L}-u_{L}\right| \\ L\left(\min \left(z_{L}, \mu_{n+1}^{L}\right), M_{n+1}\right) \\ R\left(\max \left(z_{L}, \mu_{n+1}^{L}\right), M_{n+1}\right)\end{array}\right.$ 
- $V_{R}^{\prime}\left\{\begin{array}{l}u_{R}=\frac{1}{2}\left(z_{R}+\mu_{n+1}^{R}\right) \\ H_{R}=M_{n+1}+k\left|\mu_{n+1}^{R}-u_{R}\right| \\ L\left(\min \left(z_{R}, \mu_{n+1}^{R}\right), M_{n+1}\right) \\ R\left(\max \left(z_{R}, \mu_{n+1}^{R}\right), M_{n+1}\right)\end{array}\right.$

Organize in an increasing order $V_{1}, V_{2}, \cdots, V_{n-1}, V_{L}^{\prime}, V_{R}^{\prime}: V_{1} \leq V_{2} \leq \cdots \leq V_{n} \leq V_{n+1}$.

\subsubsection{Stopping Criterion}

If $\left|H_{n+1}-\varphi_{n+1}\right| \leq \varepsilon_{0}$, then stop, else, $n=n+1$ and back to iterative steps.

\section{3. $\alpha$-Dense Curves}

The principal tool that enables one to apply the algorithm above for a multivariate function is the $\alpha$-dense curves [15] [16] [17].

\subsection{The $\alpha$-Dense Curves}

Definition 3. Let $X$ be a non empty set and $S$ a subset of $X . S$ is said to be $\alpha$-dense in $X$, if:

$$
\forall M \in X, \exists M^{\prime} \in S: d\left(M, M^{\prime}\right) \leq \alpha
$$

Among the $\alpha$-dense curves, we have chosen the Lissajous curves.

\subsection{Lissajous Curve}

In mathematics, a Lissajous curve, also known as Lissajous figure or Bowditch curve, is the graph of a system of parametric equations which describe complex harmonic motion.

\subsubsection{Bidimensional Case}

In the bidimensional case, a Lissajous figure can be defined by the following parametric equations:

$$
\left\{\begin{array}{l}
x(t)=a \sin (t) \\
y(t)=b \sin (n t+\phi)
\end{array} \text { where } 0 \leq \phi \leq \frac{\pi}{2} \text { and } n \geq 1 .\right.
$$

The number $n$ is named the parameter of the curve. If $n$ is rational, it can be expressed in the form $n=\frac{p}{q}$. Hence, the parametric equation describing the curve becomes:

$$
\left\{\begin{array}{l}
x(t)=a \sin (p t) \\
y(t)=b \sin (q t+\phi) \text { where: } 0 \leq \phi \leq \frac{\pi}{2 p} \\
0 \leq t \leq 2 \pi
\end{array}\right.
$$

In what follows, we set $\phi=0$ and let consider the following function $\Gamma$ defined by:

$$
\begin{aligned}
\Gamma:[0,2 \pi] & \rightarrow[0,2 \pi] \times[0,2 \pi] \\
t & \mapsto \Gamma(t)=\left(\Gamma_{1}(t), \Gamma_{2}(t)\right)
\end{aligned}
$$


where $\left\{\begin{array}{l}\Gamma_{1}(t)=\pi \sin (p t)+\pi \\ \Gamma_{2}(t)=\pi \sin (q t)+\pi\end{array}\right.$ such that: $p$ is an even number and $q=p+1$, of which the representative curve is given by Figure 5;

Theorem 2. If $\alpha=\pi \sin \left(\frac{\pi}{p}\right)$, the Lissajous curve $\Gamma$, given by (2), is $\alpha$-dense in $[0,2 \pi]^{2}$.

Proof. Let $M_{0}(x, y)$ any point in $[0,2 \pi]^{2}$. Let show that there exists $t \in[0,2 \pi]$ such that:

$$
d\left(M_{0}, \Gamma(t)\right) \leq \pi \sin \left(\frac{\pi}{p}\right)
$$

We Set: $\left\{\begin{array}{l}\Gamma_{1}(t)=\pi \sin (p t)+\pi \\ \Gamma_{2}(t)=\pi \sin (q t)+\pi\end{array}\right.$.

$p$ is an even number and $q=p+1$.

Let's set $t$ in $[0,2 \pi]$. Notice that the function $\Gamma_{1}$ is $\frac{2 \pi}{p}$ periodic. Let $t^{\prime}=t+\frac{2 \pi}{p}$.

Consider the points $M\left(\Gamma_{1}(t), \Gamma_{2}(t)\right)$ and $M^{\prime}\left(\Gamma_{1}\left(t^{\prime}\right), \Gamma_{2}\left(t^{\prime}\right)\right)$. The points $M$ and $M^{\prime}$ have the same abscissa.

$$
\begin{aligned}
d\left(M, M^{\prime}\right)^{2} & =\left(\Gamma_{2}\left(t^{\prime}\right)-\Gamma_{2}(t)\right)^{2}=\pi^{2}\left(\sin \left(q t^{\prime}\right)-\sin (q t)\right)^{2} \\
& =4 \pi^{2} \sin ^{2}\left(\frac{q}{2}\left(t^{\prime}-t\right)\right) \cos ^{2}\left(\frac{q}{2}\left(t+t^{\prime}\right)\right) \\
& =4 \pi^{2} \sin ^{2}\left(q \frac{\pi}{p}\right) \cos ^{2}\left(q t+q \frac{\pi}{p}\right) \\
& =4 \pi^{2} \sin ^{2}\left(\frac{\pi}{p}\right) \cos ^{2}\left(q t+\frac{\pi}{p}\right)
\end{aligned}
$$

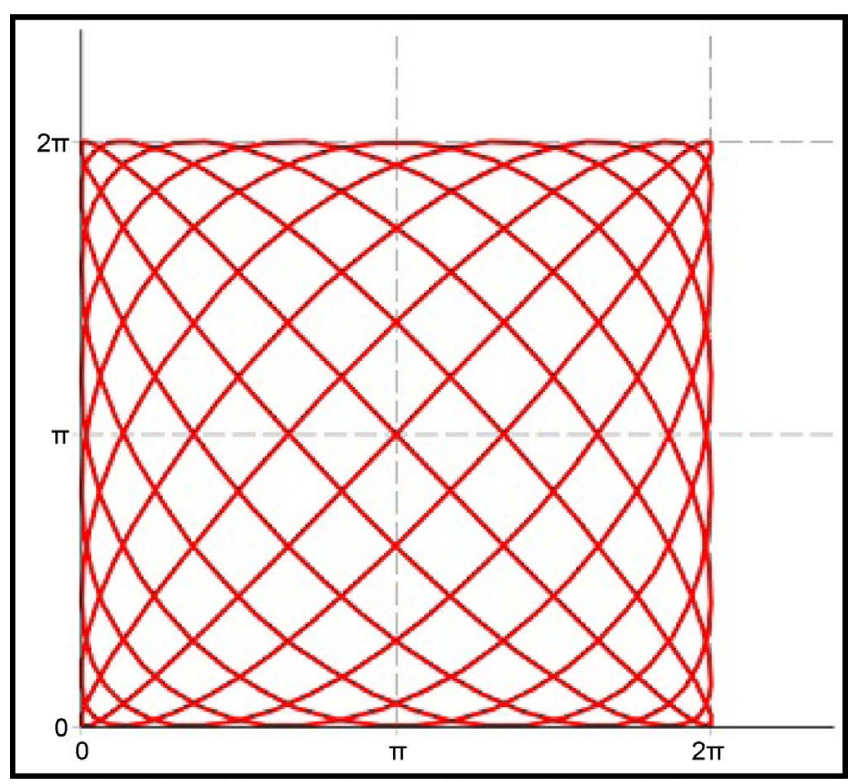

Figure 5. Lissajous curve in the bidimensional case. 
This distance reaches its maximum value when $\cos ^{2}\left(q t+\frac{\pi}{p}\right)=1$, for $t=\frac{1}{q}\left(k \pi+\frac{\pi}{p}\right)$ where $k \in\{1,2, \cdots, 2 q\}$.

Hence, $d\left(M, M^{\prime}\right) \leq 2 \pi \sin \left(\frac{\pi}{p}\right)$.

As $\Gamma_{1}$ is surjective from $\left[0, \frac{2 \pi}{p}\right]$ on $[0,2 \pi]$, there exists $t_{1} \in\left[0, \frac{2 \pi}{p}\right]$ such that $x=\Gamma_{1}\left(t_{1}\right)$.

As $\Gamma_{2}$ is surjective from $\left[0, \frac{2 \pi}{p}\right]$ on $[0,2 \pi]$, there exists $t_{2} \in\left[0, \frac{2 \pi}{p}\right]$ such that $y=\Gamma_{2}\left(t_{2}\right)$.

There exists $k \in\{0,1, \cdots, p-1\}$ such that: either

$$
\Gamma_{2}\left(t_{1}+\frac{2 k \pi}{p}\right) \leq \Gamma_{2}\left(t_{2}\right) \leq \Gamma_{2}\left(t_{1}+\frac{2(k+1) \pi}{p}\right)
$$

or

$$
\Gamma_{2}\left(t_{1}+\frac{2 k \pi}{p}\right) \geq \Gamma_{2}\left(t_{2}\right) \geq \Gamma_{2}\left(t_{1}+\frac{2(k+1) \pi}{p}\right)
$$

This does not occur only when $y=0$ or $y=2 \pi$, that means that when the point $M$ in on the boundary.

This yields that $M_{0}$ is in the segment:

$$
\left[M_{k}\left(\Gamma\left(t_{1}+\frac{2 k \pi}{p}\right)\right), M_{k+1}\left(\Gamma\left(t_{1}+\frac{2(k+1) \pi}{p}\right)\right)\right] .
$$

So that, any point $M_{0}$ can be framed between two points of type $M_{k}$ and $M_{k+1}$.

Hence, we can approximate any point of $[0,2 \pi]^{2}$ by a point of the Lissajous curve.

When trying to $\alpha$-densify $[0,2 \pi]^{2}$ using the parametric curve $\Gamma(t)$, we choose the coefficient $p$ such that:

$$
\alpha=\pi \sin \left(\frac{\pi}{p}\right) .
$$

Generally, let $a>0$ and set a curve $h$ that parametric equation is:

$$
\begin{aligned}
h:[0,2 \pi] & \rightarrow[-a, a] \times[-a, a] \\
t & \mapsto\left\{\begin{array}{l}
h_{1}(t)=\frac{a}{\pi} \Gamma_{1}(t)-a=a \sin (p t) \\
h_{2}(t)=\frac{a}{\pi} \Gamma_{2}(t)-a=a \sin (q t)
\end{array}\right.
\end{aligned}
$$

Corollary 1. For $\alpha=a \sin \left(\frac{\pi}{p}\right)$, any point in $[-a, a]^{2}$ can be approximated, with a precision $\alpha$, by at least one point of $h$.

$\forall M \in[-a, a]^{2}$, there exists $t \in[0,2 \pi]$ such that $d(M, h(t)) \leq \alpha$. 


\subsubsection{Multidimensional Case}

$\checkmark$ In dimension two, we defined the Lissajous curve by:

$$
\begin{aligned}
\Gamma:[0,2 \pi] & \rightarrow[0,2 \pi] \times[0,2 \pi] \\
t & \mapsto\left\{\begin{array}{l}
\Gamma_{1}(t)=\pi \sin (p t)+\pi \\
\Gamma_{2}(t)=\pi \sin (q t)+\pi
\end{array}\right.
\end{aligned}
$$

with: $p$ a given even number and $q=p+1$.

$\checkmark$ In dimension three, let's consider $\left(x_{1}, x_{2}, x_{3}\right) \in[0,2 \pi]^{3}$, We first link $x_{1}$ and $x_{2}$ as done in the bidimensional case: that means:

$$
x_{1}=\Gamma_{1}\left(t^{*}\right) \text { and } x_{2}=\Gamma_{2}\left(t^{*}\right)
$$

with $t^{*}$ in $[0,2 \pi]$, then we link $t^{*}$ and $x_{3}$, similarly, by setting:

$$
t^{*}=\Gamma_{1}(t) \text { and } x_{3}=\Gamma_{2}(t)
$$

with $t$ in $[0,2 \pi]$. This involves:

$$
\left\{\begin{array}{l}
x_{1}=\Gamma_{1}\left(t^{*}\right)=\Gamma_{1}\left(\Gamma_{1}(t)\right) \\
x_{2}=\Gamma_{2}\left(t^{*}\right)=\Gamma_{2}\left(\Gamma_{1}(t)\right) \\
x_{3}=\Gamma_{2}(t)
\end{array}\right.
$$

Hence, we obtain parametric curve $H(t)=\left(H_{1}(t), H_{2}(t), H_{3}(t)\right)$ defined by the following expression:

$$
\left\{\begin{array}{l}
H_{1}(t)=\Gamma_{1} \circ \Gamma_{1}(t) \\
H_{2}(t)=\Gamma_{2} \circ \Gamma_{1}(t) \\
H_{3}(t)=\Gamma_{2}(t)
\end{array}\right.
$$

with $t \in[0,2 \pi]$.

$\checkmark$ We can generalize this process to $n$ variables $\left(x_{1}, x_{2}, \cdots, x_{n}\right)$ by linking two by two by the same manner. At the end of the process, we get the new variable $t$ belonging to $[0,2 \pi]$ that all variables will be expressed by:

$$
x_{i}=H_{i}(t), i=1, \cdots, n
$$

where $H_{i}(t)$ are defined as follows:

$$
\left\{\begin{array}{l}
H_{1}(t)=\Gamma_{1}^{n-1}(t)=\underbrace{\Gamma_{1} \circ \Gamma_{1} \circ \cdots \circ \Gamma_{1}}_{n-1 \text { times }}(t) \\
H_{i}(t)=\Gamma_{2} \circ \Gamma_{1}^{n-i}(t) \forall i=2, \cdots, n
\end{array}\right.
$$

Then, let's consider the parametric curve $H$ defined by:

$$
\begin{aligned}
H:[0,2 \pi] & \rightarrow[0,2 \pi]^{n} \\
t & \mapsto\left(H_{1}(t), H_{2}(t), \cdots, H_{n}(t)\right)
\end{aligned}
$$

Theorem 3. Let $\alpha=\pi \sin \left(\frac{\pi}{p}\right)$.

The parametric curve defined by $H(t)=\left(H_{1}(t), H_{2}(t), \cdots, H_{n}(t)\right)$ such that:

$$
\left\{\begin{array}{l}
H_{1}(t)=\Gamma_{1}^{n-1}(t) \\
H_{i}(t)=\Gamma_{2} \circ \Gamma_{1}^{n-i}(t) \forall i=2, \cdots, n
\end{array}\right.
$$


for $t \in[0,2 \pi]$ is $\alpha$-dense on $[0,2 \pi]^{n}$.

Proof. Let $H(t)$ and $H\left(t+\frac{2 \pi}{p}\right)$ two points of the curve $H$. As the function $\Gamma_{1}$ is $\frac{2 \pi}{p}$ periodic, the $(n-1)$ first coordinates of these two points are equal. As proceeded in the second part of the proof of the previous theorem, we show that:

$$
d\left(H(t), H\left(t+\frac{2 \pi}{p}\right)\right) \leq 2 \pi \sin \left(\frac{\pi}{p}\right)
$$

Therefore, any point $M_{0}\left(x_{1}, x_{2}, \cdots, x_{n}\right)$ can be framed between two points of the curve of type:

$$
H\left(t+\frac{2 k \pi}{p}\right) \text { and } H\left(t+\frac{2(k+1) \pi}{p}\right) \text { where } t \in[0,2 \pi] .
$$

Generally, let $a>0$ and set a curve $h$ that parametric equation is:

$$
\begin{gathered}
h:[0,2 \pi] \rightarrow[-a, a]^{n} \\
t \mapsto\left(h_{1}(t), h_{2}(t), \cdots, h_{n}(t)\right) \\
\left\{\begin{array}{l}
h_{1}(t)=\frac{a}{\pi} \Gamma_{1}^{n-1}(t)-a \\
h_{j}(t)=\frac{a}{\pi} \Gamma_{2} \circ \Gamma_{1}^{n-j}(t)-a \text { for } j=2, \cdots, n
\end{array}\right.
\end{gathered}
$$

Corollary 2. For $\alpha=a \sin \left(\frac{\pi}{p}\right)$, any point in $[-a, a]^{n}$ can be approximated, with a precision $\alpha$, by at least one point of the parametric curve given by $h$.

$\forall M \in[-a, a]^{n}$, there exists $t \in[0,2 \pi]$ such that $d(M, h(t)) \leq \alpha$.

\section{Optimization of a Multivariate Holderian Function}

Let $f$ be a multivariate holderian function with constants of Holder are: $k>0$ and $\beta>1$.

Let us consider the following multidimensional optimization problem:

$$
\left(P_{n}\right) \underset{x \in[-a, a]^{n}}{\operatorname{Min}_{n}} f(x)
$$

In fact, we don't look for the exact value of the minimum value of $f$, we'd just want an approximating value of that minimum value with a given accuracy $\varepsilon$.

By means of an $\alpha$-dense Lissajous curve on $[-a, a]^{n}$, we convert the initial multidimensional problem $\left(P_{n}\right)$ into an unidimensional one as follows:

$$
\left(P_{1}\right) \operatorname{Min}_{t \in[0,2 \pi]} f^{*}(t)
$$

where: $f^{*}=f \circ h$, the single variable function approximating the multivariate function $f$. (where $\left(h_{1}, h_{2}, \cdots, h_{n}\right)$ defined above)

Proposition 2. If $f$ is $\left(k, \frac{1}{\beta}\right)$-holderian and $h_{i=1, n}$ is $\left(k_{i}^{\prime}, \frac{1}{\beta^{\prime}}\right)$-holderian, 
then $f^{*}=f \circ h$ is holderian where the constant is “ $k\left(\sum_{i=1}^{n} k_{i}^{\prime 2}\right)^{\frac{1}{2 \beta}}$ ” and the exponent is “ $\frac{1}{\beta \beta^{\prime}}$ ".

$$
\begin{aligned}
\left|f^{*}(x)-f^{*}(y)\right| & =|f \circ h(x)-f \circ h(y)|=|f(h(x))-f(h(y))| \\
& \leq k\|h(x)-h(y)\|^{\frac{1}{\beta}} \leq k\left(\sum_{i=1}^{n} \sqrt{\left(h_{i}(x)-h_{i}(y)\right)^{2}}\right)^{\frac{1}{\beta}}
\end{aligned}
$$

Proof.

$$
\begin{aligned}
& \leq k\left(\sum_{i=1}^{n} \sqrt{\left(k_{i}^{\prime}|x-y| \frac{1}{\beta^{\prime}}\right)^{2}}\right)^{\frac{1}{\beta}} \leq k\left(\sum_{i=1}^{n} \sqrt{k_{i}^{\prime 2}}|x-y|^{\frac{1}{\beta^{\prime}}}\right)^{\frac{1}{\beta}} \\
& \leq k\left(\sum_{i=1}^{n} k_{i}^{\prime 2}\right)^{\frac{1}{2 \beta}}|x-y|^{\frac{1}{\beta \beta^{\prime}}}, \quad t \in[0,2 \pi]
\end{aligned}
$$

Let $x_{o p t}=\arg \min (f)$ and $t_{o p t}=\arg \min \left(f^{*}\right)$.

Theorem 4. If $\alpha=\left(\frac{\varepsilon}{k}\right)^{\beta}$ then $\left|f\left(x_{\text {opt }}\right)-f^{*}\left(t_{\text {opt }}\right)\right| \leq \varepsilon$.

Remark 1. The knowledge of the minimum of $f^{*}$ allows us to surround the minimum value of $f$ in the interval $\left[f^{*}\left(t_{\text {opt }}\right)-\varepsilon, f^{*}\left(t_{\text {opt }}\right)+\varepsilon\right]$.

Proof. We set: $x_{o p t}=\arg \min _{x \in[-a, a]^{n}} f(x)$

As $x_{\text {opt }} \in[-a, a]^{n}$, the $\alpha$-density guarantees the existence of $t^{*} \in[0,2 \pi]$ such that $\left|x_{o p t}-h\left(t^{*}\right)\right| \leq \alpha$

$$
\begin{aligned}
\left|f\left(x_{o p t}\right)-f^{*}\left(t^{*}\right)\right| & =\left|f\left(x_{o p t}\right)-f\left(h\left(t^{*}\right)\right)\right| \\
& \leq k|| x_{\text {opt }}-\left.h\left(t^{*}\right)\right|^{\mid \frac{1}{\beta}} \leq k \alpha^{\frac{1}{\beta}}=\varepsilon
\end{aligned}
$$

Hence, if we want to estimate the optimum with an accuracy $\varepsilon$, we just have to take $\alpha=\left(\frac{\varepsilon}{k}\right)^{\beta}$.

Suppose that there exists $x_{0} \in[-a, a]^{n}$ such that:

$$
f\left(x_{0}\right)<f^{*}\left(t_{\text {opt }}\right)-\varepsilon
$$

So that:

$$
f\left(x_{0}\right)+\varepsilon<f^{*}\left(t_{\text {opt }}\right)
$$

The $\alpha$-densityinvolves that there exists $t_{0} \in[0,2 \pi]$ such that $\left\|x_{0}-h\left(t_{0}\right)\right\|<\alpha$

$$
\begin{gathered}
\left|f\left(x_{0}\right)-f\left(h\left(t_{0}\right)\right)\right| \leq\left. k\left\|x_{0}-h\left(t_{0}\right)\right\|\right|^{\frac{1}{\beta}} \leq \varepsilon \\
f\left(x_{0}\right)-\varepsilon \leq f\left(h\left(t_{0}\right)\right) \leq f\left(x_{0}\right)+\varepsilon
\end{gathered}
$$

Considering $(*)$ involves:

$$
f^{*}\left(t_{0}\right)=f\left(h\left(t_{0}\right)\right) \leq f\left(x_{0}\right)+\varepsilon<f^{*}\left(t_{\text {opt }}\right)
$$

This is absurd. 


\section{Numerical Tests (Figures 6-9)}

1) $f_{1}(x)=\sqrt{1-x^{2}}, x \in[-0.25,0.5]$

The holderian constants are $=\sqrt{2}, \beta=2$. The accuracy is: $\varepsilon=10^{-5}$. The result is:

$$
\left\{\begin{array}{l}
\Rightarrow x^{*}=0.5 \\
\Rightarrow f_{1}\left(x^{*}\right)=0.866 \\
f_{\text {lopt }} \in\left[f_{1}\left(x^{*}\right)-\varepsilon, f_{1}\left(x^{*}\right)\right]
\end{array}\right.
$$

2) $f_{2}(x)=\sum_{k=1}^{5} k|\sin ((3 k+1) x+k)||x-k|^{\frac{1}{5}}, x \in[0,10]$

$$
\begin{array}{r}
k=77, \beta=5, \varepsilon=0.003 \\
\left\{\begin{array}{l}
\Rightarrow x^{*}=2.829917922 \\
\Rightarrow x^{*}=2.829917922
\end{array}\right.
\end{array}
$$

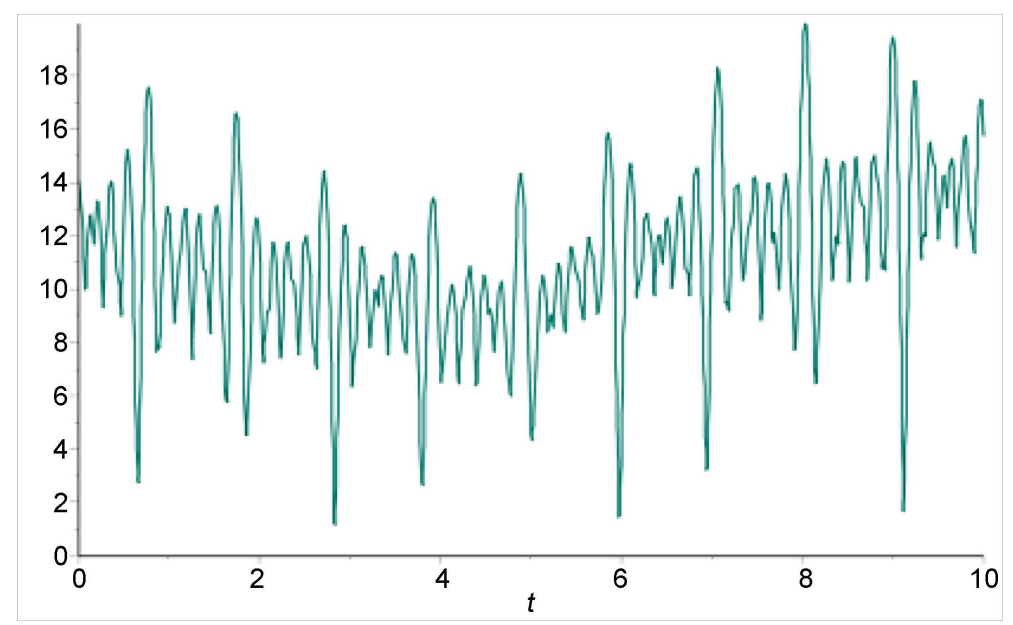

Figure 6. Curve of $f_{2}$.

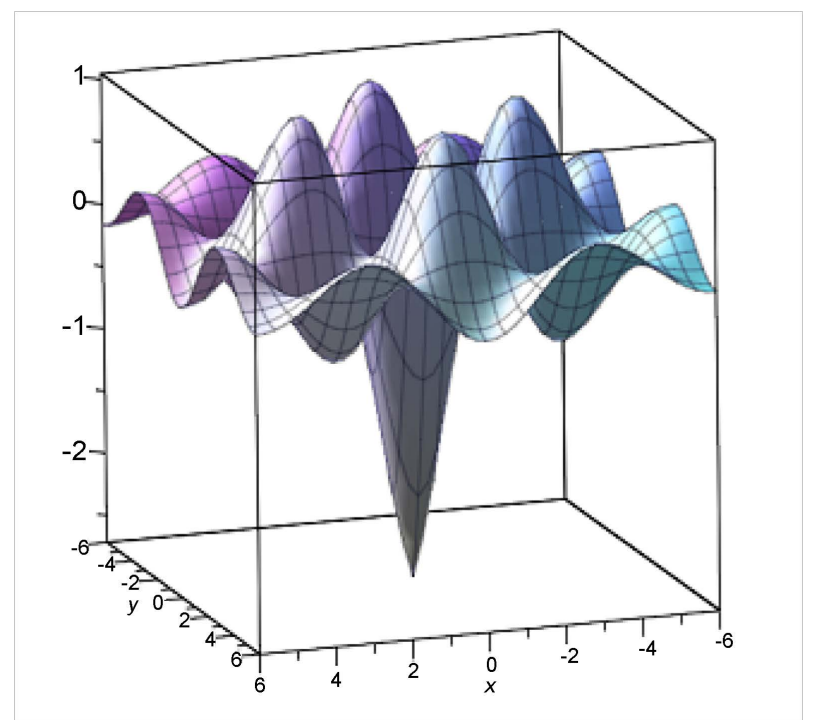

Figure 7. Curve of $f_{5}$. 


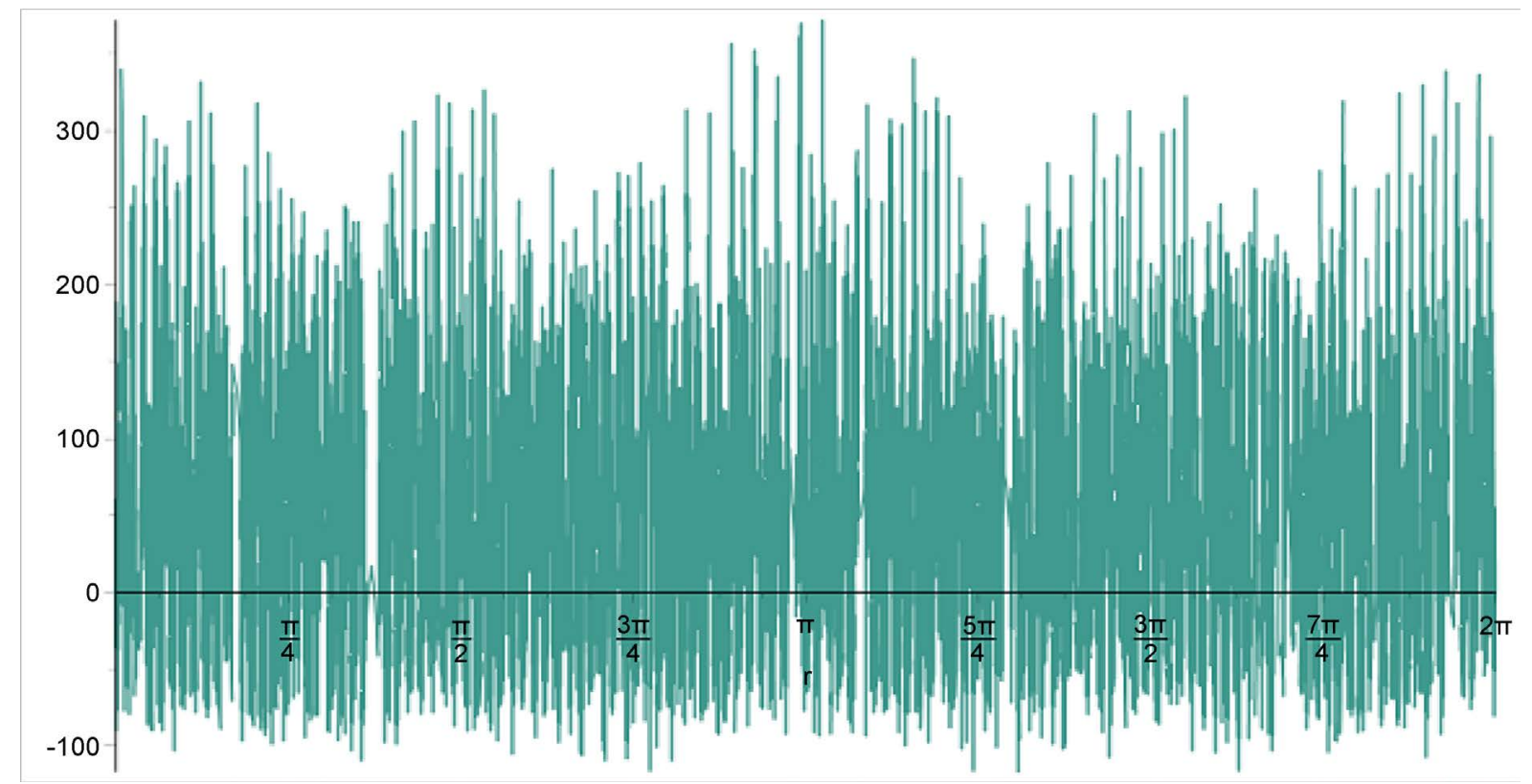

Figure 8. Curve of $f^{*}=f \circ h$.
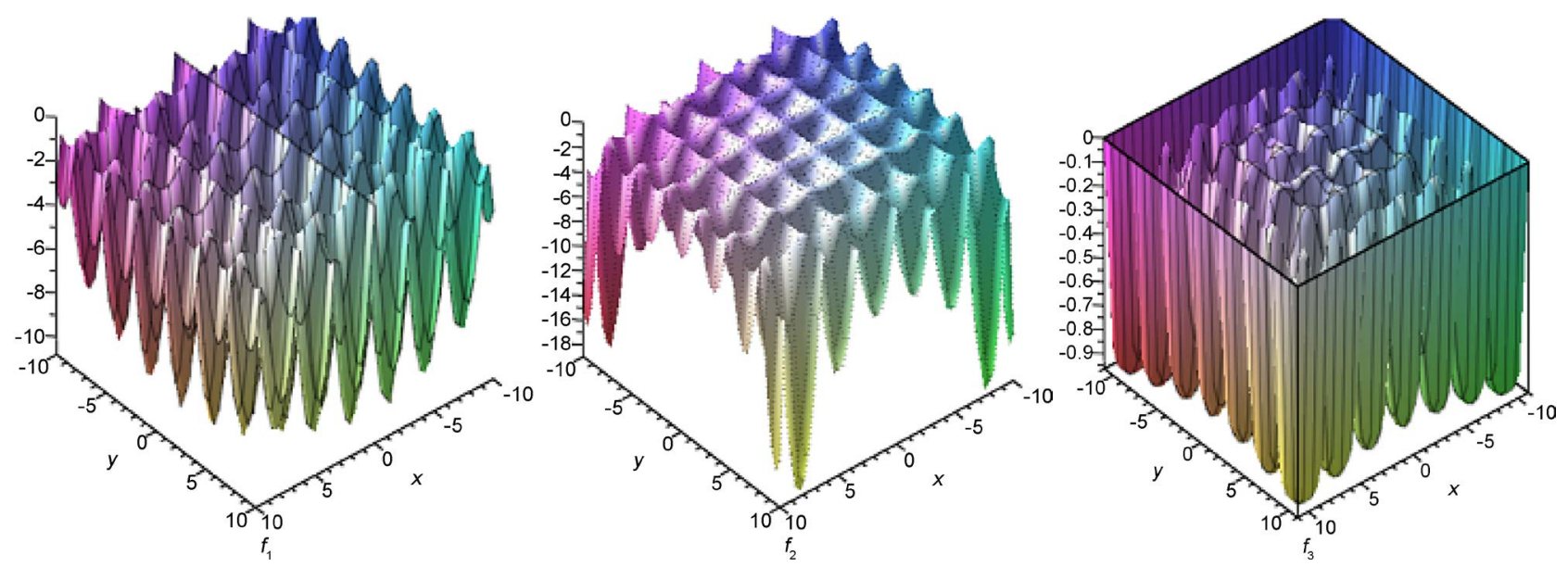

Figure 9. Curve of $f_{1}, f_{2}$ and $f_{a}$.

3) $f_{3}(x, y)=|x+y-0.25|^{\frac{2}{3}}-3 \cos \left(\frac{x}{2}\right),(x, y) \in\left[-\frac{1}{2}, \frac{1}{2}\right]^{2}$

$$
k=2.42, \beta=\frac{3}{2}, \varepsilon=0.01
$$

$$
\left\{\begin{array}{l}
\Rightarrow x^{*}=(-0.004,0.253) \\
\Rightarrow f_{3}\left(x^{*}\right)=-2.99 \\
f_{3 o p t} \in\left[f_{3}\left(x^{*}\right)-\varepsilon, f_{3}\left(x^{*}\right)\right]
\end{array}\right.
$$

4) $f_{4}(x, y)=\sum_{k=1}^{3} \frac{1}{k}\left|\cos \left(\left(\frac{3}{k}+1\right)(x+5)+\frac{1}{k}\right)\right||x-y|^{\frac{1}{3}},(x, y) \in[-5,5]^{2}$

$$
k=14.77, \beta=3, \varepsilon=0.1
$$




$$
\left\{\begin{array}{l}
\Rightarrow x^{*}=(-4.499796,-4.500100) \\
\Rightarrow f_{4}\left(x^{*}\right)=0.067788
\end{array}\right.
$$

5) $f_{5}(x, y)=-\cos x \cos y \exp \left(1-\frac{\sqrt{x^{2}+y^{2}}}{\pi}\right),(x, y) \in[-6,6]^{2}$

$$
\begin{gathered}
k=45.265, \beta=\frac{1}{2}, \varepsilon=0.03 \\
\left\{\begin{array}{l}
\Rightarrow x^{*}=(0.023391875,-0.01321677) \\
\Rightarrow f_{5}\left(x^{*}\right)=-2.694161027
\end{array}\right.
\end{gathered}
$$

6) $f_{6}\left(x_{1}, x_{2}, x_{3}\right)=\frac{1}{2} \sum_{i=1}^{3}\left(x_{i}^{4}-16 x_{i}^{2}+5 x_{i}\right),\left(x_{1}, x_{2}, x_{3}\right) \in[-5,5]^{3}$

$$
\begin{gathered}
k=180, \beta=7, \varepsilon=0.02 \\
\left\{\begin{array}{l}
\Rightarrow x^{*}=(-2.899891,-3.000102,-2.923504) \\
\Rightarrow f_{6}\left(x^{*}\right)=-117.3248028
\end{array}\right.
\end{gathered}
$$

7) Let the following functions test. In [10], RPS method was used to optimize them.

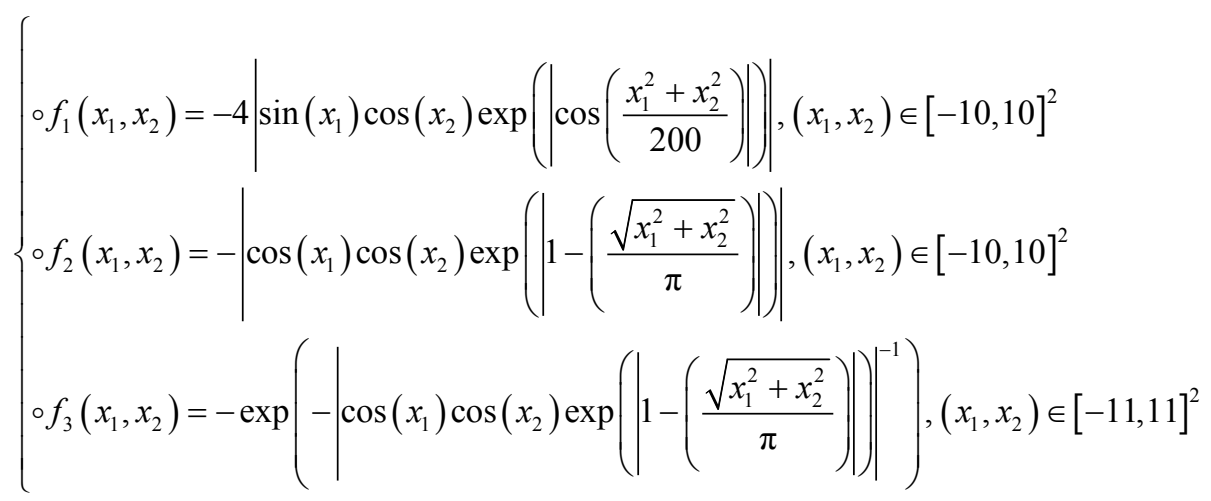

In what follows, we compare our method with the RPS one (The Particle Swarm Method of Global Optimization)

\begin{tabular}{llc}
\hline \multicolumn{3}{c}{ with under-estimator method RPS method } \\
\hline$f_{1}$ & $x^{*}=(-1.571191,0.001240), f_{1}\left(x^{*}\right)=-10.872289$ & $f_{1}\left(x^{*}\right) \simeq-10.8723$ \\
$f_{2}$ & $x^{*}=(-8.061776,9.663910), f_{2}\left(x^{*}\right)=-19.208048$ & $f_{2}\left(x^{*}\right) \simeq-19.21$ \\
$f_{3}$ & $x^{*}=(9.720125,-9.672833), f_{3}\left(x^{*}\right)=-0.963417$ & $f_{3}\left(x^{*}\right) \simeq-0.96354$ \\
\hline
\end{tabular}

\section{References}

[1] Ammar, H., Abbaoui, K. and Ndour, M. (1996) An Example of an Interaction Model between Two Species. Kybernetes, 25, 106-118.

[2] Miguel, R.L. and Sahinidis, N.V. (2013) Derivative-Free Optimization: A Review of Algorithms and Comparison of Software Implementations. Journal of Global Optimization, 56, 1247-1293. https://doi.org/10.1007/s10898-012-9951-y

[3] Lavigne, D. and Cherruault, Y. (1991) Alienor-Gabriel Global Optimization of a 
Function of Several Variables. Mathematical and Computer Modelling, 15, 125-134. https://doi.org/10.1016/0895-7177(91)90097-Q

[4] Mora, G., Cherruault, Y. and Ziadi, A. (2001) Global Optimization. A New Variant of the Alienor Method. Computers and Mathematics with Applications, 41, 63-71. https://doi.org/10.1016/S0898-1221(01)85006-9

[5] Shubert, B.O. (1972) A Sequential Method Seeking the Global Maximum of a Function. SIAM Journal on Numerical Analysis, 9, 379-388.

https://doi.org/10.1137/0709036

[6] Ammar, H. and Cherruault, Y. (1993) Approximation of Several Variables Function by a One Variable Function and Application to Global Optimization. Mathematical and Computer Modelling, 18, 17-21. https://doi.org/10.1016/0895-7177(93)90003-H

[7] Ammar, H. and Cherruault, Y. (1995) Implementation of Alienor Technique in the Multidimensional Bissection Method. Application to Global Optimization. A New Accelerated Algorithm. Kybernetes, 24, 31-40. https://doi.org/10.1108/03684929510147272

[8] Evtushenko, Ya.G., Malkova, V.U. and Stanevichyus, A.A. (2009) Parallel Global Optimization of Function of Several Variables. Computational Mathematics and Mathematical Physics, 49, 246-260. https://doi.org/10.1134/S0965542509020055

[9] Gerge, V.P. and Sergeyev, Ya.D. (1999) Sequential and Parallel Algorithms for Global Minimizing Functions with Lipschitzian Derivatives. Computers and Mathematics with Applications, 37, 163-179. https://doi.org/10.1016/S0898-1221(99)00067-X

[10] Sergeyev, Y.D. and Kvasov, D.E. (2013) Lipschitz Global Optimization Methods in Control Problems. Automation and Remote Control, 74, 1435-1448. https://doi.org/10.1134/S0005117913090014

[11] Gourdin, E., Jaumard, B. and Ellaia, R. (1996) Global Optimization of Hölder Functions. Journal of Global Optimization, 8, 323-348. https://doi.org/10.1007/BF02403997

[12] Lera, D. and Sergeyev, Ya.D. (2002) Global Minimization Algorithms for Hölder Functions. BIT Numerical Mathematics, 42, 119-133. https://doi.org/10.1023/A:1021926320198

[13] Rahal, M. and Ziadi, A. (2008) A New Extension of Piyavskii's Method to Holder Functions of Several Variables. Applied Mathematics and Computation, 197, 478488. https://doi.org/10.1016/j.amc.2007.07.067

[14] Mishra, S.K. (2007) Some New Test Functions for Global Optimization and Performance of Repulsive Particle Swarm Method. University Library of Munich, Germany, MPRA Paper 2718.

[15] Mora, G. and Cherruault, Y. (1997) Characterization and Generation of a-Dense Curve. Computers \& Mathematics with Applications, 33, 83-91. https://doi.org/10.1016/S0898-1221(97)00067-9

[16] Mora, G., Cherruault, Y. and Ziadi, A. (2000) Functional Equations Generating Space-Densifying Curves. Computers and Mathematics with Applications, 39, 4555. https://doi.org/10.1016/S0898-1221(00)00085-7

[17] Sergeyev, Y.D., Strongin, R.G. and Lera, D. (2013) Introduction to Global Optimization Exploiting Space-Filling Curves. Springer Briefs in Optimization.

https://doi.org/10.1007/978-1-4614-8042-6 
Submit or recommend next manuscript to OALib Journal and we will provide best service for you:

- Publication frequency: Monthly

- 9 subject areas of science, technology and medicine

- Fair and rigorous peer-review system

- Fast publication process

- Article promotion in various social networking sites (LinkedIn, Facebook, Twitter, etc.)

- Maximum dissemination of your research work

Submit Your Paper Online: Click Here to Submit

Or Contact service@oalib.com 